\section{Differentiated neuroblastoma F-11 cells as an alternative in-vitro model to dorsal root ganglion neurons}

\author{
Stefania Blasa, ${ }^{1}$ Valentina Pastori, ${ }^{2}$ \\ Alessia D'Aloia, ${ }^{1}$ Marzia M. Lecchi² \\ 1Department of Biotechnology and \\ Biosciences, University of Milano- \\ Bicocca, Milan; 2Department of \\ Biotechnology and Biosciences and \\ Milan Center for Neuroscience, \\ University of Milano-Bicocca, Milan, \\ Italy
}

\section{Abstract \\ We induced differentiation in F-11 cells to verify if they could show similarities with sensory neurons, in order to develop an alternative to animal models for research studies in the biomedical field.}

\section{Introduction}

Dorsal Root Ganglion (DRG) neurons have been used for years to study the mechanisms underlying the somatosensory path- ways and noxious stimuli. However, these cultures are often expensive, difficult to set up and subject to ethical issues. For these reasons, we tried to develop an alternative to animal models by using F-11 cell line, a hybridoma derived from embryonic rat DRG neurons and mouse neuroblastoma, ${ }^{1}$ whose cryopreservation and thawing are simple and economic. F-11 cells could be differentiated into functional neurons by their maintenance on biocompatible substrates, ${ }^{2}$ but their properties as sensory neurons remain so far unknown. Thus, in this work we differentiated them in serumdeprived medium in order to verify if they could develop properties of mature neurons and acquire functional similarities with DRG neurons.

\section{Materials and Methods}

Neuronal differentiation was induced by incubating F-11 cells in serum-deprived medium (1\% FBS) for 10-14 days in culture. An electrophysiological investigation by using the patch-clamp technique in the whole-cell configuration was performed for studying the generation of electrical activity and the expression of voltage-dependent $\mathrm{Na}^{+}, \mathrm{Ca}^{2+}$ and $\mathrm{K}^{+}$channels. Capsaicin, substance $\mathrm{P}$, neurotransmitters (Acetylcholine
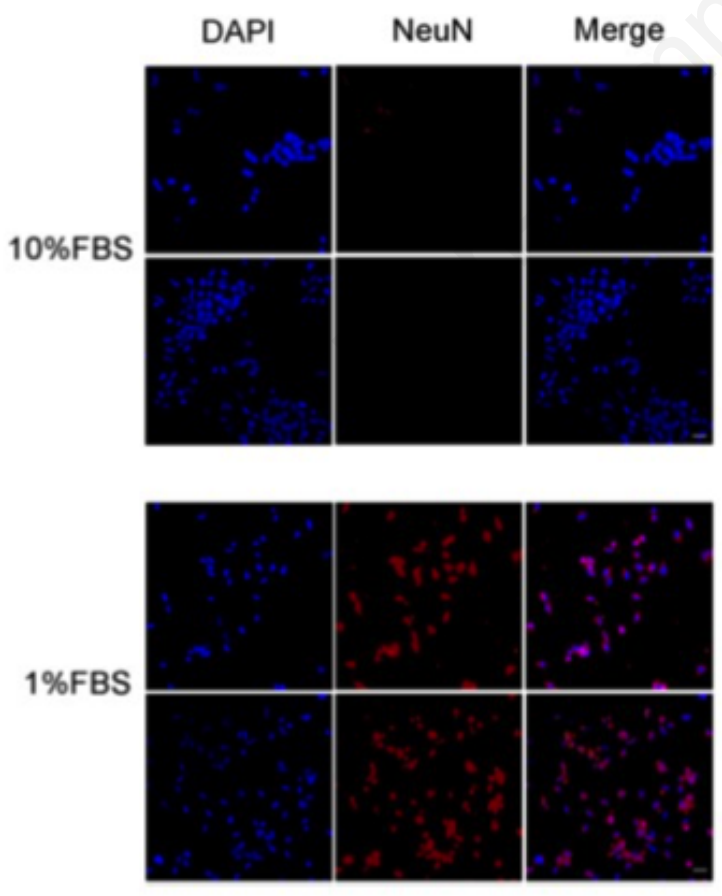

Figure 1. Differentiated F-11 cells express the neuronal nuclear antigen NeuN. The panels illustrate NeuN staining in red, DAPI in blue and the color overlay (merged) in F-11 cells maintained in 10\% FBS (control) and 1\% FBS (differentiated cells), respectively. Quantification of NeuN positive cells (histograms) in 10 different fields confirmed no or minor expression of this nuclear marker in $10 \%$ FBS compared to $1 \%$ FBS cultures.
Correspondence: Marzia M. Lecchi, Department of Biotechnology and Biosciences and Milan Center for Neuroscience, University of Milano-Bicocca, Milan, Italy.

E-mail: marzia.lecchi1@unimib.it

Key words: Sensory neurons; patch-clamp; electrophysiology; ion channels; cell biology.

Acknowledgments: We thank Dr Michela Ceriani for immunofluorescence analysis supervision. This work was funded by Fondo di Ateneo per la Ricerca (FARgrant) from the University of Milano-Bicocca and by Fondo per il finanziamento delle attività base di ricerca (FFABR) from Italian Ministry of Education, University and Research to Marzia Lecchi. The funders had no role in study design, data collection and analysis, decision to publish, or preparation of the manuscript

Disclosures: The authors declare that they have nothing to disclose nor competing interests.

Conference presentation: This paper was presented at the Third Centro 3R Annual Meeting - L'era delle 3R: modelli in silico, in vitro e in vivo per promuovere la ricerca traslazionale 30 September - 1 October 2021, Evento online organizzato dal Politecnico di Torino.

Received for publication: 9 July 2021.

Accepted for publication: 7 September 2021.

This work is licensed under a Creative Commons Attribution NonCommercial 4.0 License (CC BY-NC 4.0).

CCopyright: the Author(s), 2021

Licensee PAGEPress, Italy

Biomedical Science and Engineering 2021; 4(s1):153 doi:10.4081/bse.2021.153

and Glutamate) and acidic solutions were used to verify the expression of ion channels typical of sensory neurons. Data were presented as mean \pm s.e.m. Mean comparisons between the properties of differentiated and undifferentiated cells (10\% serum) were obtained by using the unpaired $t$-test or the non-parametric Mann-Whitney test. The number of responsive cells in the two conditions was compared by using the $\chi^{2}$ test. The significance level was set for $\mathrm{p}<0,05$.

\section{Results}

After 12-14 days in 1\% FBS medium, F-11 cells showed properties of mature neurons: they stained positively for the neuronal nuclear protein NeuN (Figure 1) and 

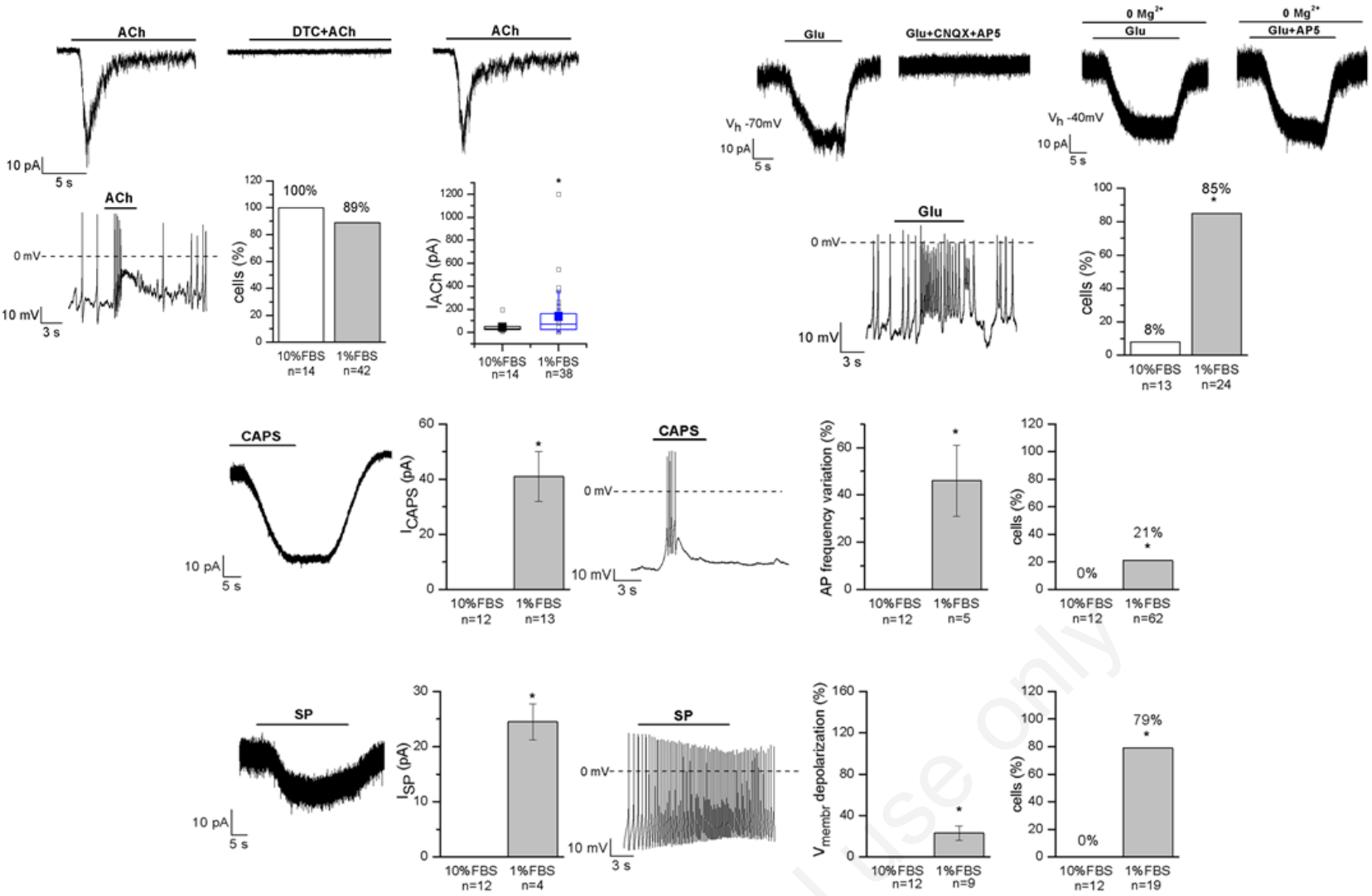

Figure 2. Differentiated F-11 cells display responses to Acetylcholine (ACh), Glutamate (Glu), Capsaicin and Substance P. ACh was effective on $100 \%$ of undifferentiated and on $89 \%$ of differentiated cells and its action was mediated by nAChRs, as demonstrated by d-tubocurarine (DTC) block. The percentage of Glu-responsive cells was significantly higher in differentiated than in undifferentiated cells. Glu-evoked effects were principally mediated by non-NMDA receptors, since AP5 administration in $\mathrm{Mg}_{2+}$-free extracellular solution and at $-40 \mathrm{mV}$ did not affect them. Capsaicin (CAPS) and Substance P (SP) evoked responses in differentiated cells but had no effect on undifferentiated cells.

about $50 \%$ of the culture was characterized by neuronal networks of cells exhibiting typical neuronal morphology. Moreover, differentiated F-11 cells showed $\mathrm{Na}^{+}$currents consistent with those exhibited by primary DRG neurons. Responses to acetylcholine (ACh) were recorded in $89 \%$ of differentiated cells. Approximately the same percentage $(70-80 \%)$ of rat DRG primary neurons was referred by literature to express functional nicotinic $\mathrm{ACh}$ receptors (nAChRs). Glutamate (Glu) was effective on non-NMDA receptors as in embryonic DRG neurons (Figure 2). Moreover, pH 5 induced desensitizing currents through proton-activated channels also expressed in DRG neurons. Responses to capsaicin and substance $\mathrm{P}$ were also recorded in $21 \%$ and $79 \%$ of differentiated cells respectively.

\section{Discussion and Conclusions}

Dissociated DRG neurons represent the ideal model for investigating sensory neurons, but their availability is limited by several issues. Therefore, the use of immortalized cell lines is considered a valid alternative to animal models. We demonstrated that differentiated F-11 cells represent a more accessible, simple and less expensive model compared to DRG neurons and, since they express some ion channels and receptors that are also expressed in sensory neurons, they might be employed for studying mechanisms involved in the detection and transmission of noxious stimuli and sensory inflammatory pain. ${ }^{3}$

\section{References}

1. Platika D, Boulos MH, Baizer L, Fishman MC. Neuronal traits of clonal cell lines derived by fusion of dorsal root ganglia neurons with neuroblastoma cells. Proc Natl Acad Sci USA 1985;82:3499-503.

2. Russo L, Sgambato A, Lecchi M, et al. Neoglucosylated collagen matrices drive neuronal cells to differentiate. ACS Chem Neurosci. 2014;5:261-5.

3. Yu Y-Q, Chen, X-F, Yang Y, et al. Electrophysiological identification of tonic and phasic neurons in sensory dorsal root ganglion and their distinct implications in inflammatory pain. Physiol Res 2014;63:793-9. 